\title{
Motivos y barreras para hacer ejercicio y practicar deportes en Madrid
}

\author{
Gabriel Rodríguez-Romo, ${ }^{1}$ Carlos Boned-Pascual ${ }^{2}$ \\ y María Garrido-Muñoz ${ }^{2}$
}

Forma de citar

Rodríguez-Romo G, Boned-Pascual C, Garrido-Muñoz M. Motivos y barreras para hacer ejercicio y practicar deportes en Madrid. Rev Panam Salud Publica. 2009;26(3):244-54.

RESUMEN Objetivos. Analizar los motivos y barreras de la población metropolitana de Madrid para realizar actividad física y/o deporte, así como sus relaciones con el género, la edad y la posición social.

Métodos. Estudio transversal realizado con una muestra de 625 personas, con datos representativos a nivel global, con un margen de error del $\pm 4 \%$ y un intervalo de confianza del 95,5\%. El tipo de muestreo fue polietápico, utilizándose un cuestionario ad hoc.

Resultados. Diversión (29,9\%), mantenimiento de la forma $(26,4 \%)$ y salud $(16,1 \%)$, fueron los motivos de práctica más frecuentes, mostrando diferencias según género $\left[\chi^{2}(8)=19,635\right.$; $\mathrm{p}=0,009 ;$ IC95\%: 0,007-0,011] y edad $\left[\chi^{2}(32)=55,671 ; \mathrm{p}=0,006 ;\right.$ IC95\%:0,005-0,008]. Respecto al abandono de la práctica, las exigencias laborales y familiares $(24,1 \%)$ y la falta de tiempo (22,2\%), fueron las causas más habituales, relacionándose con la edad $\left[\chi^{2}(24)=36,955\right.$; $\mathrm{p}=0,041 ;$ IC95\%: 0,037-0,044] y marginalmente con el género $\left[\chi^{2}(6)=11,753 ; \mathrm{p}=0,069 ;\right.$ IC95\%: 0,063-0,074]. Los motivos más frecuentes para no haber practicado nunca fueron la falta de tiempo $(24,4 \%)$ y el hecho de no gustarles $(24,4 \%)$, no existiendo relaciones con las variables analizadas.

Conclusiones. La práctica físico-deportiva en la muestra estudiada tiene un carácter lúdico y recreativo y está dirigida al mantenimiento y mejora de la salud y a la adquisición de un buen aspecto físico. Los motivos que conducen a la práctica y a su abandono están relacionados con la edad y el género. En cambio, las razones para no haber practicado nunca son independientes de las variables analizadas.

Palabras clave Actividad física; ejercicio; motivación; deportes; estilo de vida; conducta de salud; España.

Uno de los objetivos de salud de todas las sociedades avanzadas es aumentar la actividad física, desarrollando políticas y estrategias efectivas encaminadas tanto a

\footnotetext{
Facultad de Ciencias de la Actividad Física y del Deporte, Universidad Politécnica de Madrid. La correspondencia debe dirigirse a: Gabriel RodríguezRomo, Universidad Politécnica de Madrid, Facultad de Ciencias de la Actividad Física y del Deporte, C/Martín Fierro 7, 28040, Madrid, España. Correo electrónico: gabriel.rodriguez@upm.es

2 Facultad de Ciencias de la Actividad Física y del Deporte, Universidad Europea de Madrid, España.
}

estimularla como a suprimir las barreras que la impiden. Como base para el diseño de dichas políticas y estrategias, diversos autores han manifestado la necesidad de conocer los patrones de actividad física de la población, así como los factores y los motivos que llevan a las personas a iniciarla, a mantenerla o a abandonarla (1-3).

Está claro que las razones que llevan a las personas a hacer ejercicio o abandonarlo poseen un carácter dinámico y raramente se concretan en un solo motivo.
Además, estudios recientes sobre muestras heterogéneas de gran tamaño prueban que dichas razones difieren según los grupos de población considerados (4-6). Tales diferencias resultan particularmente evidentes cuando se analizan los motivos y las barreras para la práctica en función de la edad y del género, aun cuando algunos trabajos hallan diferencias asociadas también a otras variables, tales como el tipo de actividad y la frecuencia con que se practica $(4,7)$ y el nivel sociocultural de las personas (5). 
Según un estudio realizado a una muestra de estudiantes universitarios de Barcelona en 2007 (8), las barreras parecen vincularse en mayor medida con aspectos de la situación individual que atraviesa la persona en cada momento, mientras que las motivaciones se presentan más estables y se relacionan con el estilo de vida que lleva la persona.

En cuanto a las razones que llevan a realizar ejercicio, diversos trabajos señalan que las mujeres adultas tienden más a manifestar motivaciones estéticas, como cuidar el peso y en general mejorar su imagen física $(6,7,9,10)$, mientras que los hombres buscan desarrollar habilidades y destrezas relacionadas con la competición y con la diversión $(5,6,11)$. Asimismo, las mujeres mayores también parecen señalar motivos de componente más social que los hombres (12) como, por ejemplo, el apoyo de familiares y amigos (13). Además, entre las mujeres jóvenes universitarias son más frecuentes las razones de índole intelectual y social, como aprender cosas nuevas o la búsqueda de nuevas amistades (11).

Si bien los jóvenes de ambos sexos informan que el disfrute y la diversión son uno de sus principales estímulos para hacer ejercicio $(5,6)$, otros autores encontraron que en este grupo de población la actividad física tiene como propósitos cuidar la apariencia física (el peso corporal en las mujeres, el desarrollo muscular en los hombres) y ampliar su red de contactos sociales y el apoyo familiar (14). A medida que aumenta la edad, sin embargo, estas razones físicas y estéticas van cediendo importancia en favor de otras, como descubrir cosas nuevas, reducir el estrés y evadir la rutina diaria (6, $7,11)$. Igualmente, la salud y el sentirse mejor adquieren progresivamente una mayor gravitación hasta convertirse en las razones prioritarias de los adultos mayores $(2,7,15)$. Dicho esto, en estudios de una amplia muestra de niños y jóvenes españoles otros autores encontraron también motivos relacionados con la salud (16).

En su decisión de hacer ejercicios físicos, las personas de más edad también aducen razones de corte social, como el apoyo de familiares y amigos y la posibilidad de interactuar con otras personas, además de la recomendación médica (6, 12, 14, 17).

En cuanto a la posición social, un estudio desarrollado con una muestra representativa de la población española pone de manifiesto que entre las personas que señalan ocupar una posición social más desahogada, la evasión y el escapar de la rutina habitual, junto con el gusto por el deporte y la ejercitación física, adquieren más importancia como motivos de práctica que en el resto de grupos sociales. En cambio, entre las clases sociales más desfavorecidas, destaca en mayor medida la posibilidad de encontrarse con amigos (5).

En todo caso, las razones que motivan a hacer ejercicio o practicar deportes, así como las que llevan a abandonarlos, conllevan numerosos factores relacionados con las circunstancias personales y familiares de los individuos más que con la pertenencia a un determinado estrato social (5).

Así, en comparación con los hombres, las mujeres suelen aducir un mayor número de razones para no practicar actividades físicas $(2,18)$. Los hombres de edad avanzada argumentan falta de motivación de manera más habitual que las mujeres, aun cuando las mujeres aluden más a menudo malestar y aspectos relacionados con la falta de apoyo social (12, 19), en particular de sus familiares, y desaprobación de su pareja (20). También se halló que entre las barreras más fuertes entre las mujeres figuran la falta de tiempo y de motivación (9).

Según la edad, las barreras manifestadas con mayor frecuencia por los más jóvenes son las experiencias negativas relacionadas con la práctica de actividad física en el colegio, la falta de roles de referencia y el escaso apoyo por parte de la pareja (14). En la población adulta en general, las barreras más comunes suelen ser el no tener tiempo $(1,6,21)$, carencia de instalaciones, pérdida de interés (6, $21,22) y$, aunque en menor medida, no tener compañeros o dinero (22), salud pobre, dolores (23), el cuidado de los hijos (1) o experiencias negativas en el pasado (14). En el caso de los adultos mayores, el no hacer ejercicio fue atribuido fundamentalmente a razones relacionadas con la salud, como no tener condiciones físicas, incapacidad, presencia de dolores $(1,2,12,15,24)$ y miedo a sufrir caídas $(24,25)$.

La amplia diversidad de razones, motivos y desestímulos que presentan las personas para iniciar, mantener o abandonar la práctica de actividad física ha llevado a sugerir, desde diversas perspectivas, la necesidad de elaborar estrategias individuales o dirigidas a grupos específicos como la forma más efectiva de incidir en la reducción de la inactividad de determinados sectores de la población $(6,24,26,27)$.

En el caso concreto de la región de Madrid, hasta fechas recientes no se había llevado a cabo ningún estudio específico y representativo enfocado a analizar las actitudes e intereses de su población en relación con la actividad física y el deporte. Por ello, a lo largo de 2005 se implementó un estudio en esta línea (28). A partir de dicho estudio, en el presente trabajo se analizan los motivos y las barreras percibidas por la población del área metropolitana de Madrid para hacer ejercicios o practicar deportes en su tiempo libre. Se examinan asimismo las posibles relaciones existentes entre dichos motivos y barreras y algunas de las variables sociodemográficas que parecen tener una mayor influencia en las decisiones de los madrileños en este respecto, incluidos el género, la edad y la posición social (29). El conocimiento del peso de estos motivos y barreras podría servir de base para desarrollar políticas y estrategias dirigidas a promover la actividad física y el deporte entre los habitantes $y$, de manera especial, entre aquellos grupos de población en los que se registran mayores tasas de inactividad, es decir los adultos, y en particular los mayores, las mujeres y las personas de nivel socioeconómico medio-bajo o bajo (28).

\section{MATERIALES Y MÉTODOS}

Se realizó un estudio de tipo descriptivo con diseño de corte transversal, utilizándose el cuestionario administrado mediante entrevista personal como técnica de recogida de datos. La población objeto de estudio fueron personas con edades comprendidas entre 15 y 69 años que residían en el área metropolitana de Madrid, es decir, en el municipio de Madrid y en las Coronas Metropolitanas Norte, Sur, Este y Oeste (cuadro 1). El universo del muestreo ascendía a un total de $3677635^{3}$ personas. De dicha población se extrajo una muestra de 625 participantes, lo que permitió obtener datos representativos con un intervalo de confianza de $95,5 \%$ y un margen de error de $\pm 4 \%$, suponiendo que $p=q$ para la situación más desfavorable. La afijación de la muestra fue proporcional

\footnotetext{
Datos del Instituto Nacional de Estadística (INE) de España, 2005.
} 
CUADRO 1. Características generales de la muestra estudiada $(n=625)$, Madrid, España, 2005

\begin{tabular}{lrr}
\hline \multicolumn{1}{c}{ Característica } & No. & $\%$ \\
\hline Género & & \\
Hombres & 301 & 48,2 \\
Mujeres & 324 & 51,8 \\
Edad (años) & 68 & 10,9 \\
$15-19$ & 194 & 31,0 \\
$20-29$ & 135 & 21,6 \\
$30-44$ & 145 & 23,2 \\
$45-59$ & 83 & 13,3 \\
$60-69$ & & \\
Posición social & 12 & 1,9 \\
Alta & 130 & 20,8 \\
Media-alta & 334 & 53,4 \\
Media & 87 & 13,9 \\
Media-baja & 35 & 5,6 \\
Baja & 27 & 4,3 \\
No sabe/No contesta & & \\
Nivel de práctica & 348 & 55,7 \\
Practica actividad física y/o deporte en el tiempo libre & 158 & 25,3 \\
Ha practicado actividad física y/o deporte anteriormente, pero ahora no & 119 & 19,0 \\
Nunca ha practicado actividad física ni deporte en el tiempo libre & & \\
Area geográfica & 372 & 59,5 \\
Municipio de Madrid (Madrid Capital) & 28 & 4,5 \\
Corona Metropolitana Norte & 60 & 9,6 \\
Corona Metropolitana Este & 132 & 21,1 \\
Corona Metropolitana Sur & 33 & 5,3 \\
Corona Metropolitana Oeste & & \\
\hline
\end{tabular}

al número de habitantes en cada una de las áreas geográficas consideradas, así como a la distribución de la población objeto de estudio según género y grupos de edad.

La selección de las personas que integraron la muestra se llevó a cabo mediante un muestreo polietápico. La selección de las unidades de primera etapa (municipios integrantes de las diferentes Coronas Metropolitanas y distritos del municipio de Madrid) en las que se administraron los cuestionarios, se realizó de manera intencional, tratando de lograr una adecuada dispersión de la muestra entre las áreas mencionadas. Las unidades de la segunda etapa (lugar concreto de inicio de cada ruta aleatoria en el correspondiente municipio o distrito) se eligieron al azar. Posteriormente, fueron los entrevistadores participantes en el estudio quienes seleccionaron al azar a las personas que serían entrevistadas, siguiendo las instrucciones y cuotas que previamente se les había facilitado.

La recogida de datos se efectuó mediante un cuestionario elaborado ad hoc, cuya construcción se basó primordialmente en la selección y la adaptación de las preguntas ya realizadas en otros estudios sobre hábitos deportivos, tanto en el ámbito europeo (30) como nacional (31) y regional $(32,33)$. Tras la primera elaboración formal, el cuestionario fue sometido al examen de diversos expertos, quienes proporcionaron recomendaciones y comentarios de valor. El cuestionario preliminar se aplicó de manera experimental a un pequeño grupo de personas para valorar la idoneidad y claridad - comprensión- de sus preguntas $y$, finalmente, se produjo la versión definitiva (publicada en referencia 28).

La pregunta inicial del cuestionario permitía que los entrevistados se posicionaran en su nivel de actividad física o deporte, en una de las siguientes categorías: 1) Practico actividad física $y / o$ deporte en mi tiempo libre; 2) He practicado actividad física y/o deporte anteriormente, pero ahora no; 3) Nunca he practicado actividad física ni deporte en mi tiempo libre. A partir de las respuestas, se procedió a analizar los motivos y barreras para la práctica de ejercicios físicos en el tiempo libre, planteándose para ello tres preguntas cerradas. Las preguntas, y las opciones de respuesta, fueron en gran medida una adaptación de las ya empleadas en la encuesta nacional sobre hábitos deportivos (31), a fin de obtener resultados comparables con el conjunto de la población española.

Así, solamente a las personas que declararon realizar actividad física o deporte en su tiempo libre se les preguntó la razón o el motivo principal por el que lo hacían, incluyéndose su respuesta en alguna de las siguientes categorías: por diversión y por pasar el tiempo; por relacionarme con amigos; porque me gusta y me agrada; por mantenerme en forma o mantener la línea; por mantener o mejorar la salud; por competir y mejorar mi rendimiento deportivo; por evasión y por salir de lo habitual; por otras razones. Únicamente a aquellos participantes que señalaron haber abandonado de manera temporal o permanente la actividad física o el deporte en el tiempo libre, se les preguntó la causa principal de haberlo hecho, incluyéndose la falta de instalaciones cercanas o adecuadas; lesiones o problemas de salud; edad inadecuada; exigencias derivadas de los estudios o del trabajo; pereza o desgana; no tener el hábito; carencia de apoyo y estímulo (amigos, pareja, padres) o de recursos económicos; obligaciones familiares; falta de tiempo; otras causas. Finalmente, sólo a las personas que manifestaron no haber realizado nunca actividad física ni deporte en su tiempo libre se les pidió que expusieran la razón principal que justificaba dicha conducta. En las opciones de respuesta, además de las indicadas en el caso del abandono, se incluyó también la siguiente: no me gusta, no le veo beneficios ni utilidad.

En cuanto a las variables sociodemográficas (género, edad y posición social) objeto de análisis en este estudio por su posible relación con los motivos y las barreras para hacer ejercicio físico o practicar deportes, cabe señalar que la posición social se determinó a partir de la percepción personal de los propios entrevistados que, en función de su nivel de ingresos propios o familiares, debían situarse en alguna de las siguientes categorías: alta, media-alta, media, mediabaja o baja. Se consideró que esta alternativa era más adecuada que preguntar directamente por otros indicadores objetivos, ya que los cuestionarios se administraron mediante entrevista personal.

Todas las entrevistas, cuya duración osciló entre 10 y 20 minutos, fueron efectuadas entre marzo y junio de 2005 por entrevistadores previamente capacitados para su correcta ejecución. Asimismo, durante la realización del trabajo de campo, se llevaron a cabo las correspondientes tareas de coordinación y control.

\section{Análisis estadístico}

Con los datos obtenidos de las entrevistas se efectuaron análisis descriptivos de 
las tres variables objeto de estudio (motivos para realizar ejercicio o practicar deportes en el tiempo libre, razones para su abandono y razones para no haberlo hecho nunca) en función de las características sociodemográficas de los entrevistados (género, edad y posición social), calculándose también en todos los casos los intervalos de confianza de $95 \%$. Con objeto de comprobar las posibles relaciones de los motivos y barreras con las variables sociodemográficas consideradas, se llevaron a cabo pruebas no paramétricas de la $\chi^{2}$, utilizándose la técnica de Monte Carlo para la estimación no sesgada del nivel de significación e informándose de este y de su correspondiente intervalo de confianza al $95 \%$. A fin de determinar las combinaciones entre las que existían diferencias significativas, se efectuaron análisis de residuos tipificados. Los puntos de corte fueron los valores de $Z$ correspondientes a los niveles de significa- ción $0,05( \pm 1,96)$ y $0,01( \pm 2,57)$. Todos los análisis fueron realizados mediante el paquete estadístico SPSS, versión 15.0.

\section{RESULTADOS}

Las características generales de la muestra estudiada se recogen en el cuadro 1. Como puede apreciarse, de las 625 personas entrevistadas, 51,8\% eran mujeres y $48,2 \%$, hombres. Por edades, el grupo de participantes más numeroso era el de 20 a 29 años (31\%), seguido por los de 45 y 59 años (23\%) y los de entre 30 y 44 años (21,6\%). Algo más de la mitad de los entrevistados $(53,4 \%)$ consideraba ocupar una posición social media y casi $60 \%$ de la muestra residía en el municipio de Madrid. En cuanto al nivel de práctica de actividad física o deporte en el tiempo libre, 55,7\% de la muestra manifestó realizar, con mayor o menor frecuencia, algún tipo de ejercicio o deporte en su tiempo libre, $25,3 \%$ señaló haber abandonado, de manera temporal o permanente, y 19\% indicó no haber hecho nunca actividad física ni deportes en su tiempo libre.

De los 348 encuestados que declararon realizar algún tipo de actividad física o deporte en su tiempo libre, la mayoría (29\%) mencionó la diversión y la ocupación amena del tiempo libre como el motivo principal, seguido por el mantenimiento de la forma y de la línea $(26,4 \%)$, el mantenimiento o la mejora de la salud $(16,1 \%)$, el gusto por el deporte y las sensaciones agradables relacionadas con la práctica $(14,9 \%)$, el permanecer en contacto con los amigos $(5,2 \%)$, la competición y la mejora del rendimiento deportivo $(3,2 \%)$, la evasión o el escaparse de lo habitual $(1,4 \%)$ y otros motivos no incluidos en los anteriores $(1,4 \%)$ (cuadros 2 y 3 ).

Las pruebas de la $\chi^{2}$ efectuadas para analizar la posible relación entre los mo-

CUADRO 2. Motivo principal para la práctica de actividad física y/o deporte en el tiempo libre, según género y edad. Área metropolitana de Madrid, España, 2005

\begin{tabular}{|c|c|c|c|c|c|c|c|c|}
\hline \multirow[b]{2}{*}{ Motivo } & \multicolumn{2}{|c|}{ Género } & \multicolumn{6}{|c|}{ Edad (años) } \\
\hline & $\begin{array}{c}\text { Hombres } \\
(n=193) \\
\% \\
\left(^{\text {IC95\%) }}{ }^{\mathrm{a}}\right. \\
\text { Residuos }^{\mathrm{b}}\end{array}$ & $\begin{array}{c}\text { Mujeres } \\
(n=155) \\
\% \\
(I C 95 \%) \\
\text { Residuos }\end{array}$ & $\begin{array}{c}15-19 \\
(n=48) \\
\% \\
\text { (IC95\%) } \\
\text { Residuos }\end{array}$ & $\begin{array}{c}20-29 \\
(n=128) \\
\% \\
(I C 95 \%) \\
\text { Residuos }\end{array}$ & $\begin{array}{c}30-44 \\
(n=75) \\
\% \\
\text { (IC95\%) } \\
\text { Residuos }\end{array}$ & $\begin{array}{c}45-59 \\
(n=68) \\
\% \\
\text { (IC95\%) } \\
\text { Residuos }\end{array}$ & $\begin{array}{c}60-69 \\
(n=29) \\
\% \\
(I C 95 \%) \\
\text { Residuos }\end{array}$ & $\begin{array}{c}\text { Total } \\
(n=348) \\
\% \\
(I C 95 \%)\end{array}$ \\
\hline Diversión, pasar el tiempo & $\begin{array}{c}37,8 \\
(31,0-44,6) \\
3,6\end{array}$ & $\begin{array}{c}20,0 \\
(13,7-26,3) \\
-3,6\end{array}$ & $\begin{array}{c}50,0 \\
(35,9-64,1) \\
3,3\end{array}$ & $\begin{array}{c}32,0 \\
(23,9-40,1) \\
0,7\end{array}$ & $\begin{array}{c}26,7 \\
(16,7-36,7) \\
-0,7\end{array}$ & $\begin{array}{c}17,6 \\
(8,5-26,7) \\
-2,5\end{array}$ & $\begin{array}{c}24,1 \\
(8,5-39,7) \\
-0,7\end{array}$ & $\begin{array}{c}29,9 \\
(25,1-34,7)\end{array}$ \\
\hline Mantenerse en forma, mantener la línea & $\begin{array}{c}23,8 \\
(17,8-29,8) \\
-1,2\end{array}$ & $\begin{array}{c}29,7 \\
(22,5-36,9) \\
1,2\end{array}$ & $\begin{array}{c}8,3 \\
(0,5-16,1) \\
-3,1\end{array}$ & $\begin{array}{c}28,1 \\
(20,3-35,9) \\
0,5\end{array}$ & $\begin{array}{c}26,7 \\
(16,7-36,7) \\
0,1\end{array}$ & $\begin{array}{c}38,2 \\
(26,7-49,7) \\
2,5\end{array}$ & $\begin{array}{c}20,7 \\
(6,0-35,4) \\
-0,7\end{array}$ & $\begin{array}{c}26,4 \\
(21,8-31,0)\end{array}$ \\
\hline Mantener o mejorar la salud & $\begin{array}{c}14,0 \\
(9,1-18,9) \\
-1,2\end{array}$ & $\begin{array}{c}18,7 \\
(12,6-24,8) \\
1,2\end{array}$ & $\begin{array}{c}14,6 \\
(4,6-24,6) \\
-0,3\end{array}$ & $\begin{array}{c}11,7 \\
(6,1-17,3) \\
-1,7\end{array}$ & $\begin{array}{c}14,7 \\
(6,7-22,7) \\
-0,4\end{array}$ & $\begin{array}{c}17,6 \\
(8,5-26,7) \\
0,4\end{array}$ & $\begin{array}{c}37,9 \\
(20,2-55,6) \\
3,3\end{array}$ & $\begin{array}{c}16,1 \\
(12,2-20,0)\end{array}$ \\
\hline Le gusta, le agrada & $\begin{array}{c}13,0 \\
(8,3-17,7) \\
-1,2\end{array}$ & $\begin{array}{c}17,4 \\
(11,4-23,4) \\
1,2\end{array}$ & $\begin{array}{c}12,5 \\
(3,1-21,9) \\
-0,5\end{array}$ & $\begin{array}{c}13,3 \\
(7,4-19,2) \\
-0,7\end{array}$ & $\begin{array}{c}17,3 \\
(8,7-25,9) \\
0,7\end{array}$ & $\begin{array}{c}17,6 \\
(8,5-26,7) \\
0,7\end{array}$ & $\begin{array}{c}13,8 \\
(1,2-26,4) \\
-0,2\end{array}$ & $\begin{array}{c}14,9 \\
(11,2-18,6)\end{array}$ \\
\hline Relacionarse con amigos & $\begin{array}{c}5,2 \\
(2,1-8,3) \\
0,0\end{array}$ & $\begin{array}{c}5,2 \\
(1,7-8,7) \\
0,0\end{array}$ & $\begin{array}{c}2,1 \\
(0,0-6,2) \\
-1,0\end{array}$ & $\begin{array}{c}7,0 \\
(2,6-11,4) \\
1,2\end{array}$ & $\begin{array}{c}5,3 \\
(0,2-10,4) \\
0,1\end{array}$ & $\begin{array}{c}5,9 \\
(0,3-11,5) \\
0,3\end{array}$ & $\begin{array}{c}0,0 \\
\text { NA } \\
-1,3\end{array}$ & $\begin{array}{c}5,2 \\
(2,9-7,5)\end{array}$ \\
\hline Competir y mejorar el rendimiento deportivo & $\begin{array}{c}3,6 \\
(1,0-6,2) \\
0,6\end{array}$ & $\begin{array}{c}2,6 \\
(0,1-5,1) \\
-0,6\end{array}$ & $\begin{array}{c}2,1 \\
(0,0-6,2) \\
-0,5\end{array}$ & $\begin{array}{c}6,3 \\
(2,1-10,5) \\
2,5\end{array}$ & $\begin{array}{c}2,7 \\
(0,0-6,4) \\
-0,3\end{array}$ & $\begin{array}{c}0,0 \\
\text { NA } \\
-1,7\end{array}$ & $\begin{array}{c}0,0 \\
\text { NA } \\
-1,0\end{array}$ & $\begin{array}{c}3,2 \\
(1,4-5,0)\end{array}$ \\
\hline Evasión, salir de lo habitual & $\begin{array}{c}0,0 \\
\text { NA } \\
-2,5\end{array}$ & $\begin{array}{c}3,2 \\
(0,4-6,0) \\
2,5\end{array}$ & $\begin{array}{c}2,1 \\
(0,0-6,2) \\
0,4\end{array}$ & $\begin{array}{c}1,6 \\
(0,0-3,8) \\
0,2\end{array}$ & $\begin{array}{c}1,3 \\
(0,0-3,9) \\
-0,1\end{array}$ & $\begin{array}{c}1,5 \\
(0,0-4,4) \\
0,0\end{array}$ & $\begin{array}{c}0,0 \\
\text { NA } \\
-0,7\end{array}$ & $\begin{array}{c}1,4 \\
(0,2-2,6)\end{array}$ \\
\hline Otros & $\begin{array}{c}1,0 \\
(0,0-2,4) \\
-0,7\end{array}$ & $\begin{array}{c}1,9 \\
(0,0-4,0) \\
0,7\end{array}$ & $\begin{array}{c}4,2 \\
(0,0-9,9) \\
1,7\end{array}$ & $\begin{array}{c}0,0 \\
\text { NA } \\
-1,7\end{array}$ & $\begin{array}{c}2,7 \\
(0,0-6,4) \\
1,0\end{array}$ & $\begin{array}{c}1,5 \\
(0,0-4,4) \\
0,0\end{array}$ & $\begin{array}{c}0,0 \\
\text { NA } \\
-0,7\end{array}$ & $\begin{array}{c}1,4 \\
(0,2-2,6)\end{array}$ \\
\hline No sabe / No contesta & $\begin{array}{c}1,6 \\
(0,0-3,4) \\
0,2\end{array}$ & $\begin{array}{c}1,3 \\
(0,0-3,1) \\
-0,2\end{array}$ & $\begin{array}{c}4,2 \\
(0,0-9,9) \\
1,7\end{array}$ & $\begin{array}{c}0,0 \\
\text { NA } \\
-1,7\end{array}$ & $\begin{array}{c}2,7 \\
(0,0-6,4) \\
1,0\end{array}$ & $\begin{array}{c}0,0 \\
\text { NA } \\
-1,1\end{array}$ & $\begin{array}{c}3,4 \\
(0,0-10,0) \\
1,0\end{array}$ & $\begin{array}{c}1,4 \\
(0,2-2,6)\end{array}$ \\
\hline
\end{tabular}

a Intervalo de confianza de $95 \%$.

${ }^{\mathrm{b}}$ Residuos tipificados corregidos. 
CUADRO 3. Motivo principal para la práctica de actividad física y/o deporte en el tiempo libre, según posición social. Área metropolitana de Madrid, España, 2005

\begin{tabular}{|c|c|c|c|c|}
\hline \multirow[b]{2}{*}{ Motivo } & \multicolumn{4}{|c|}{ Posición social } \\
\hline & $\begin{array}{c}\text { Alta/Media-alta } \\
\qquad \begin{array}{c}n=86) \\
\% \\
(I C 95 \%)^{\mathrm{a}}\end{array}\end{array}$ & $\begin{array}{c}\text { Media } \\
(n=202) \\
\% \\
(I C 95 \%)\end{array}$ & $\begin{array}{c}\text { Media-baja/Baja } \\
(n=49) \\
\% \\
(I C 95 \%)\end{array}$ & $\begin{array}{c}\text { Total } \\
(n=348) \\
\% \\
(I C 95 \%)\end{array}$ \\
\hline Diversión, pasar el tiempo & $\begin{array}{c}23,3 \\
(14,4-32,2)\end{array}$ & $\begin{array}{c}34,2 \\
(27,7-40,7)\end{array}$ & $\begin{array}{c}26,5 \\
(14,1-38,9)\end{array}$ & $\begin{array}{c}29,9 \\
(25,1-34,7)\end{array}$ \\
\hline Mantenerse en forma, mantener la línea & $\begin{array}{c}38,4 \\
(28,1-48,7)\end{array}$ & $\begin{array}{c}22,8 \\
(17,0-28,6)\end{array}$ & $\begin{array}{c}22,4 \\
(10,7-34,1)\end{array}$ & $\begin{array}{c}26,4 \\
(21,8-31,0)\end{array}$ \\
\hline Mantener o mejorar la salud & $\begin{array}{c}12,8 \\
(5,7-19,9)\end{array}$ & $\begin{array}{c}16,8 \\
(11,6-22,0)\end{array}$ & $\begin{array}{c}16,3 \\
(6,0-26,6)\end{array}$ & $\begin{array}{c}16,1 \\
(12,2-20,0)\end{array}$ \\
\hline Le gusta, le agrada & $\begin{array}{c}16,3 \\
(8,5-24,1)\end{array}$ & $\begin{array}{c}12,9 \\
(8,3-17,5)\end{array}$ & $\begin{array}{c}18,4 \\
(7,6-29,2)\end{array}$ & $\begin{array}{c}14,9 \\
(11,2-18,6)\end{array}$ \\
\hline Relacionarse con amigos & $\begin{array}{c}5,8 \\
(0,9-10,7)\end{array}$ & $\begin{array}{c}5,0 \\
(2,0-8,0)\end{array}$ & $\begin{array}{c}6,1 \\
(0,0-12,8)\end{array}$ & $\begin{array}{c}5,2 \\
(2,9-7,5)\end{array}$ \\
\hline Competir y mejorar el rendimiento deportivo & $\begin{array}{c}2,3 \\
(0,0-5,5)\end{array}$ & $\begin{array}{c}3,5 \\
(1,0-6,0)\end{array}$ & $\begin{array}{c}4,1 \\
(0,0-9,7)\end{array}$ & $\begin{array}{c}3,2 \\
(1,4-5,0)\end{array}$ \\
\hline Por evasión, por salir de lo habitual & $\begin{array}{l}0,0 \\
\text { NA }\end{array}$ & $\begin{array}{c}1,5 \\
(0,0-3,2)\end{array}$ & $\begin{array}{c}2,0 \\
(0,0-5,9)\end{array}$ & $\begin{array}{c}1,4 \\
(0,2-2,6)\end{array}$ \\
\hline Otros & $\begin{array}{l}0,0 \\
\text { NA }\end{array}$ & $\begin{array}{c}2,0 \\
(0,1-3,9)\end{array}$ & $\begin{array}{c}2,0 \\
(0,0-5,9)\end{array}$ & $\begin{array}{c}1,4 \\
(0,2-2,6)\end{array}$ \\
\hline No sabe/No contesta & $\begin{array}{c}1,2 \\
(0,0-3,5)\end{array}$ & $\begin{array}{c}1,5 \\
(0,0-3,2)\end{array}$ & $\begin{array}{c}2,0 \\
(0,0-5,9)\end{array}$ & $\begin{array}{c}1,4 \\
(0,2-2,6)\end{array}$ \\
\hline
\end{tabular}

a Intervalo de confianza de $95 \%$.

tivos principales que conducen a la práctica y las variables sociodemográficas objeto de estudio revelaron que dichos motivos mostraban diferencias estadísticamente significativas en función del género $\left[\chi^{2}(8)=19,635 ; p=0,009\right.$; IC95\%: $0,007-0,011]$ y de la edad $\left[\chi^{2}(32)=55,671\right.$; $p=0,006$; IC95\%: 0,005-0,008] de los practicantes, aunque no según su posición social $\left[\chi^{2}(16)=14,074 ; p=0,594\right.$; IC95\%: 0,584-0,603].

En el caso del género, en el cuadro 2 se observa que la diversión y la ocupación amena del tiempo libre $(37,8 \%)$, así como el mantener la forma y la línea $(23,8 \%)$, eran los motivos principales de práctica señalados con mayor frecuencia por los hombres, y también por las mujeres, aunque en orden inverso, situándose en primer lugar el mantenimiento de la forma y de la línea $(29,7 \%)$. El análisis de los residuos tipificados mostró que las diferencias halladas en los motivos principales de práctica en función del género se debían a que la frecuencia de hombres que declaraban realizar actividad física o deporte por diversión y por ocupar el tiempo libre era superior a la esperada por azar $(p<0,01)$, mientras que la de aquellos que señalaron la evasión o el escapar de lo habitual como principal motivo de práctica resultó infe- rior a la esperada $(p<0,05)$. En el caso de las mujeres ocurría justo al contrario.

En cuanto a la edad, en el cuadro 2 se puede apreciar que la diversión y la ocupación del tiempo libre era la primera razón por la que realizaban actividad física o deporte los grupos de población más jóvenes, con $50 \%$ los de 15 a 19 años de edad y $32 \%$ los de 20 a 29 años. Le seguían el mantenimiento y la mejora de la salud en el caso de los adolescentes (1519 años), con 14,6\% de las respuestas, y el mantenimiento de la forma y de la línea, con 28,1\% para los participantes que tenían entre 20 y 29 años. En cambio, para los siguientes grupos de población de más edad (30-44 años y 45-59 años), el mantenimiento de la forma y de la línea era el motivo primordial (26,7\% y $38,2 \%$ respectivamente). Le seguían la diversión y la ocupación del tiempo libre entre los participantes de 30 a 44 años (en una proporción idéntica del 26,7\%) y el mantenimiento y la mejora de la salud, la diversión y el gusto por el deporte (con el 17,6\% de las respuestas en los tres casos) entre los entrevistados de 45 a 59 años. Para los entrevistados de mayor edad (60 a 69 años) que hacían ejercicio, el mantenimiento o la mejora de la salud era el motivo más mencionado $(37,9 \%)$, seguido por la diversión y la ocupación amena del tiempo libre $(24,1 \%)$, el mantenimiento de la forma y de la línea $(20,7 \%)$ y las sensaciones agradables relacionadas con la propia práctica $(13,8 \%)$.

Pues bien, las relaciones encontradas entre la edad y los motivos principales para hacer ejercicio al efectuar las pruebas de la $\chi^{2}$, obedecían a que la frecuencia de adolescentes (15 a 19 años) que practicaban principalmente por diversión y ocupación del tiempo libre fue significativamente superior a la esperada $(p<0,01)$, mientras que la de los que practicaban por mantenerse en forma o mantener la línea resultó inferior a la esperada $(p<0,01)$. Estas relaciones se daban de manera inversa en los practicantes que tenían entre 45 y 59 años ( $p<0,05$ en ambos casos). Asimismo, la proporción de entrevistados con edades comprendidas entre los 60 y los 69 años que declaró que hacía ejercicio principalmente para mantener o mejorar la salud, también fue significativamente superior a la esperada por azar $(p<0,01)$.

Considerando la posición social de los participantes, ya se ha indicado que no se encontraron relaciones entre esta variable y los motivos argumentados por los entrevistados para realizar actividad física o 
practicar deportes en su tiempo libre. No obstante, a nivel descriptivo puede apreciarse que el mantenimiento de la forma y de la línea era el motivo de práctica señalado con mayor frecuencia por aquellos que consideraban ocupar una posición social media-alta o alta $(38,4 \%)$, seguido por la diversión y la ocupación del tiempo $(23,3 \%)$. En cambio, para los entrevistados que indicaron ocupar una posición social media, media-baja o baja, la diversión era el motivo de práctica más habitual, con el mantenimiento de la forma y de la línea en segundo lugar (cuadro 3).

Respecto a la razón principal que condujo a abandonar temporal o permanentemente la actividad física o el deporte a 158 de los encuestados (el 25,3\% de la muestra), las exigencias derivadas de los estudios o del trabajo y las obligaciones familiares $(24,1 \%)$ eran la causa mencionada más a menudo, aunque seguida muy de cerca por la falta de tiempo $(22,2 \%)$. A mayor distancia, otras razones que también se daban frecuentemente para justificar esta conducta eran los problemas de salud y las lesiones $(15,8 \%)$, la edad inadecuada $(10,1 \%)$, la falta de apoyo, estímulo y recursos $(8,2 \%)$ y la pereza, la desgana y la falta de hábito $(7,6 \%)$ (cuadros 4 y 5).

Los análisis efectuados revelaron la existencia de una relación marginalmente significativa entre las razones argumentadas para el abandono de la práctica y el género de los entrevistados $\left[\chi^{2}(6)=11,753\right.$; $p=0,069$; IC95\%: 0,063$0,074]$. Como muestra el cuadro 4 , las exigencias derivadas de los estudios o del trabajo, la falta de tiempo, los problemas de salud y la edad inadecuada eran, en este orden, las razones más frecuentes para el abandono de la práctica, tanto en los hombres como en las mujeres. Sin embargo, el porcentaje de hombres que identificó la edad como un impedimento para continuar practicando fue significativamente superior al esperado por azar, mientras que en las mujeres resultó inferior $(p<0,01)$.
Asimismo, en el cuadro 4 puede observarse que entre los más jóvenes (15-19 y 20-29 años) la falta de tiempo representaba la causa más frecuente para el abandono $(42,9 \%$ y $30,4 \%$ respectivamente), disminuyendo su peso como razón principal para cesar la práctica a medida que aumentaba la edad de los entrevistados. En el caso de los participantes con edades de entre 20 y 29 años, junto a la falta de tiempo también aparecían las exigencias derivadas de los estudios o del trabajo y las obligaciones familiares como otra causa de abandono bastante frecuente $(23,9 \%)$, en lo cual ( $\sin$ contar la falta de tiempo) coincidían con el resto de los grupos de edad (30 a 69 años). Empero, en los entrevistados de 30 a 44 años las razones subsecuentes de abandono del ejercicio o el deporte eran, por un lado, la falta de tiempo y, por otro, la falta de apoyo, de estímulo y de recursos, mientras que para los participantes de más edad (45 a 69 años) los problemas de salud y las lesiones, junto

CUADRO 4. Razón principal para el abandono de la práctica de actividad física y/o deporte en el tiempo libre, según género y edad. Área metropolitana de Madrid, España, 2005

\begin{tabular}{|c|c|c|c|c|c|c|c|c|}
\hline \multirow[b]{2}{*}{ Razón } & \multicolumn{2}{|c|}{ Género } & \multicolumn{6}{|c|}{ Edad (años) } \\
\hline & $\begin{array}{c}\text { Hombres } \\
(n=76) \\
\% \\
\text { (IC95\%) }^{\mathrm{a}} \\
\text { Residuos }^{\mathrm{b}}\end{array}$ & $\begin{array}{c}\text { Mujeres } \\
(n=82) \\
\% \\
(I C 95 \%) \\
\text { Residuos }\end{array}$ & $\begin{array}{c}15-19 \\
(n=14) \\
\% \\
\text { (IC95\%) } \\
\text { Residuos }\end{array}$ & $\begin{array}{c}20-29 \\
(n=46) \\
\% \\
\text { (IC95\%) } \\
\text { Residuos }\end{array}$ & $\begin{array}{c}\text { 30-44 } \\
(n=38) \\
\% \\
\text { (IC95\%) } \\
\text { Residuos }\end{array}$ & $\begin{array}{c}45-59 \\
(n=40) \\
\% \\
\text { (IC95\%) } \\
\text { Residuos }\end{array}$ & $\begin{array}{c}60-69 \\
(n=20) \\
\% \\
(I C 95 \%) \\
\text { Residuos }\end{array}$ & $\begin{array}{c}\text { Total } \\
(n=158) \\
\% \\
(\mathrm{IC} 95 \%)\end{array}$ \\
\hline $\begin{array}{l}\text { Exigencias derivadas de los estudios } \\
\text { o del trabajo, obligaciones familiares }\end{array}$ & $\begin{array}{c}25,0 \\
(15,3-34,7) \\
0,2\end{array}$ & $\begin{array}{c}23,2 \\
(14,1-32,3) \\
-0,2\end{array}$ & $\begin{array}{l}0,0 \\
\mathrm{NA} \\
-2,2\end{array}$ & $\begin{array}{c}23,9 \\
(11,6-36,2) \\
-0,1\end{array}$ & $\begin{array}{c}28,9 \\
(14,5-43,3) \\
0,8\end{array}$ & $\begin{array}{c}22,5 \\
(9,6-35,4) \\
-0,2\end{array}$ & $\begin{array}{c}35,0 \\
(14,1-55,9) \\
1,2\end{array}$ & $\begin{array}{c}24,1 \\
(17,4-30,8)\end{array}$ \\
\hline Falta de tiempo & $\begin{array}{c}18,4 \\
(9,7-27,1) \\
-1,1\end{array}$ & $\begin{array}{c}25,6 \\
(16,2-35,0) \\
1,1\end{array}$ & $\begin{array}{c}42,9 \\
(24,9-60,9) \\
2,0\end{array}$ & $\begin{array}{c}30,4 \\
(17,1-43,7) \\
1,6\end{array}$ & $\begin{array}{c}21,1 \\
(8,1-34,1) \\
-0,2\end{array}$ & $\begin{array}{c}12,5 \\
(2,3-22,7) \\
-1,6\end{array}$ & $\begin{array}{c}10,0 \\
(0,0-23,1) \\
-1,5\end{array}$ & $\begin{array}{c}22,2 \\
(15,7-28,7)\end{array}$ \\
\hline Problemas de salud, lesiones & $\begin{array}{c}17,1 \\
(8,6-25,6) \\
0,4\end{array}$ & $\begin{array}{c}14,6 \\
(7,0-22,2) \\
-0,4\end{array}$ & $\begin{array}{c}7,1 \\
(0,0-16,4) \\
-0,9\end{array}$ & $\begin{array}{c}17,4 \\
(6,4-28,4) \\
0,3\end{array}$ & $\begin{array}{c}10,5 \\
(0,8-20,2) \\
-1,0\end{array}$ & $\begin{array}{c}17,5 \\
(5,7-29,3) \\
0,4\end{array}$ & $\begin{array}{c}25,0 \\
(6,0-44,0) \\
1,2\end{array}$ & $\begin{array}{c}15,8 \\
(10,1-21,5)\end{array}$ \\
\hline Edad inadecuada & $\begin{array}{c}17,1 \\
(8,6-25,6) \\
2,8\end{array}$ & $\begin{array}{c}3,7 \\
(0,0-7,8) \\
-2,8\end{array}$ & $\begin{array}{c}14,3 \\
(1,6-27,0) \\
0,5\end{array}$ & $\begin{array}{l}0,0 \\
\text { NA } \\
-2,7\end{array}$ & $\begin{array}{c}5,3 \\
(0,0-12,4) \\
-1,2\end{array}$ & $\begin{array}{c}20,0 \\
(7,6-32,4) \\
2,5\end{array}$ & $\begin{array}{c}20,0 \\
(2,5-37,5) \\
1,6\end{array}$ & $\begin{array}{c}10,1 \\
(5,4-14,8)\end{array}$ \\
\hline $\begin{array}{l}\text { Falta de apoyo, estímulo, recursos, } \\
\text { instalaciones }\end{array}$ & $\begin{array}{c}5,3 \\
(0,3-10,3) \\
-1,3\end{array}$ & $\begin{array}{c}11,0 \\
(4,2-17,8) \\
1,3\end{array}$ & $\begin{array}{c}14,3 \\
(1,6-27,0) \\
0,9\end{array}$ & $\begin{array}{c}4,3 \\
(0,0-10,2) \\
-1,2\end{array}$ & $\begin{array}{c}13,2 \\
(2,4-24,0) \\
1,3\end{array}$ & $\begin{array}{c}7,5 \\
(0,0-15,7) \\
-0,2\end{array}$ & $\begin{array}{c}5,0 \\
(0,0-14,6) \\
-0,6\end{array}$ & $\begin{array}{c}8,2 \\
(3,9-12,5)\end{array}$ \\
\hline Pereza y desgana, falta de hábito & $\begin{array}{c}7,9 \\
(1,8-14,0) \\
0,1\end{array}$ & $\begin{array}{c}7,3 \\
(1,7-12,9) \\
-0,1\end{array}$ & $\begin{array}{c}7,1 \\
(0,0-16,4) \\
-0,1\end{array}$ & $\begin{array}{c}15,2 \\
(4,8-25,6) \\
2,3\end{array}$ & $\begin{array}{c}7,9 \\
(0,0-16,5) \\
0,1\end{array}$ & $\begin{array}{c}2,5 \\
(0,0-7,3) \\
-1,4\end{array}$ & $\begin{array}{c}0,0 \\
\text { NA } \\
-1,4\end{array}$ & $\begin{array}{c}7,6 \\
(3,5-11,7)\end{array}$ \\
\hline Otras & $\begin{array}{c}9,2 \\
(2,7-15,7) \\
-0,9\end{array}$ & $\begin{array}{c}13,4 \\
(6,0-20,8) \\
0,9\end{array}$ & $\begin{array}{c}14,3 \\
(1,6-27,0) \\
0,3\end{array}$ & $\begin{array}{c}8,7 \\
(0,6-16,8) \\
-0,7\end{array}$ & $\begin{array}{c}13,2 \\
(2,4-24,0) \\
0,4\end{array}$ & $\begin{array}{c}15,0 \\
(3,9-26,1) \\
0,9\end{array}$ & $\begin{array}{c}5,0 \\
(0,0-14,6) \\
-1,0\end{array}$ & $\begin{array}{c}11,4 \\
(6,4-16,4)\end{array}$ \\
\hline No sabe/No contesta & $\begin{array}{c}0,0 \\
\text { NA } \\
\ldots\end{array}$ & $\begin{array}{c}1,2 \\
(0,0-3,6) \\
\ldots\end{array}$ & $\begin{array}{c}0,0 \\
\text { NA } \\
\ldots\end{array}$ & $\begin{array}{c}0,0 \\
\text { NA } \\
\cdots\end{array}$ & $\begin{array}{c}0,0 \\
\text { NA } \\
\cdots\end{array}$ & $\begin{array}{c}2,5 \\
(0,0-7,3) \\
\ldots\end{array}$ & $\begin{array}{c}0,0 \\
\text { NA } \\
\ldots\end{array}$ & $\begin{array}{c}0,6 \\
(0,0-1,8)\end{array}$ \\
\hline
\end{tabular}

a Intervalo de confianza de $95 \%$.

${ }^{\mathrm{b}}$ Residuos tipificados corregidos. 
CUADRO 5. Razón principal para el abandono de la práctica de actividad física y/o deporte en el tiempo libre, según posición social. Área metropolitana de Madrid, España, 2005

\begin{tabular}{|c|c|c|c|c|}
\hline \multirow[b]{2}{*}{ Razón } & \multicolumn{4}{|c|}{ Posición social } \\
\hline & $\begin{array}{c}\text { Alta/Media-alta } \\
(n=35) \\
\% \\
(\text { IC } 95 \%)^{\mathrm{a}}\end{array}$ & $\begin{array}{c}\text { Media } \\
(n=73) \\
\% \\
(I C 95 \%)\end{array}$ & $\begin{array}{c}\text { Media-baja/Baja } \\
(n=41) \\
\% \\
(\mathrm{IC} 95 \%)\end{array}$ & $\begin{array}{c}\text { Total } \\
(n=158) \\
\% \\
(I C 95 \%)\end{array}$ \\
\hline $\begin{array}{l}\text { Exigencias derivadas de los estudios } \\
\text { o del trabajo, obligaciones familiares }\end{array}$ & $\begin{array}{c}34,3 \\
(18,6-50,0)\end{array}$ & $\begin{array}{c}17,8 \\
(9,0-26,6)\end{array}$ & $\begin{array}{c}26,8 \\
(13,2-40,4)\end{array}$ & $\begin{array}{c}24,1 \\
(17,4-30,8)\end{array}$ \\
\hline Falta de tiempo & $\begin{array}{c}11,4 \\
(0,9-21,9)\end{array}$ & $\begin{array}{c}28,8 \\
(18,4-39,2)\end{array}$ & $\begin{array}{c}19,5 \\
(7,4-31,6)\end{array}$ & $\begin{array}{c}22,2 \\
(15,7-28,7)\end{array}$ \\
\hline Problemas de salud, lesiones & $\begin{array}{c}20,0 \\
(6,7-33,3)\end{array}$ & $\begin{array}{c}11,0 \\
(3,8-18,2)\end{array}$ & $\begin{array}{c}17,1 \\
(5,6-28,6)\end{array}$ & $\begin{array}{c}15,8 \\
(10,1-21,5)\end{array}$ \\
\hline Edad inadecuada & $\begin{array}{c}2,9 \\
(0,0-8,5)\end{array}$ & $\begin{array}{c}12,3 \\
(4,8-19,8)\end{array}$ & $\begin{array}{c}12,2 \\
(2,2-22,2)\end{array}$ & $\begin{array}{c}10,1 \\
(5,4-14,8)\end{array}$ \\
\hline $\begin{array}{l}\text { Falta de apoyo, estímulos, recursos, } \\
\text { instalaciones }\end{array}$ & $\begin{array}{c}8,6 \\
(0,0-17,9)\end{array}$ & $\begin{array}{c}11,0 \\
(3,8-18,2)\end{array}$ & $\begin{array}{c}2,4 \\
(0,0-7,1)\end{array}$ & $\begin{array}{c}8,2 \\
(3,9-12,5)\end{array}$ \\
\hline Pereza y desgana, falta de hábito & $\begin{array}{c}11,4 \\
(0,9-21,9)\end{array}$ & $\begin{array}{c}6,8 \\
(1,0-12,6)\end{array}$ & $\begin{array}{c}7,3 \\
(0,0-15,3)\end{array}$ & $\begin{array}{c}7,6 \\
(3,5-11,7)\end{array}$ \\
\hline Otras & $\begin{array}{c}11,4 \\
(0,9-21,9)\end{array}$ & $\begin{array}{c}11,0 \\
(3,8-18,2)\end{array}$ & $\begin{array}{c}14,6 \\
(3,8-25,4)\end{array}$ & $\begin{array}{c}11,4 \\
(6,4-16,4)\end{array}$ \\
\hline No sabe/No contesta & $\begin{array}{l}0,0 \\
\text { NA }\end{array}$ & $\begin{array}{c}1,4 \\
(0,0-4,1)\end{array}$ & $\begin{array}{l}0,0 \\
\text { NA }\end{array}$ & $\begin{array}{c}0,6 \\
(0,0-1,8)\end{array}$ \\
\hline
\end{tabular}

a Intervalo de confianza de $95 \%$.

con la edad inadecuada, eran las siguientes causas citadas con más frecuencia.

De cualquier manera, las relaciones encontradas entre la edad de los entrevistados y las razones aducidas para el abandono de la práctica $\left[\chi^{2}(24)=36,955\right.$; $p=0,041$; IC95\%: 0,037-0,044] obedecían, por un lado, a que los entrevistados de menor edad (15 a 19 años) señalaron, en una proporción significativamente superior a la esperada por azar $(p<0,05)$, que la falta de tiempo era la principal razón para dicho abandono, aun cuando las exigencias derivadas de los estudios o del trabajo aparecían en una proporción inferior a la esperada $(p<0,05)$. Más todavía, el análisis de los residuos tipificados mostró que el porcentaje de personas con edades comprendidas entre los $45 \mathrm{y}$ los 59 años que indicaron haber abandonado la práctica de actividad física o el deporte por considerar que tenían una edad inadecuada era significativamente superior al esperado $(p<0,01)$, mientras que en los participantes de 20 a 29 años resultó ser inferior $(p<0,01)$. Además, en este último grupo de edad, la pereza y la desgana y la falta de hábito, aunque ocupaba la cuarta posición en orden de importancia entre los motivos para el abandono, también apareció con una frecuencia superior a la esperada por azar $(p<0,05)$.
Las razones principales de abandono de la práctica expuestas sí fueron independientes de la posición social que creían ocupar los entrevistados $\left[\chi^{2}(12)=13,403\right.$; $p=0,352$; IC95\%: 0,343-0,361]. De cualquier modo, a nivel descriptivo puede apreciarse que las exigencias derivadas de los estudios o del trabajo y las obligaciones familiares eran el motivo más habitual para abandonar la práctica entre los participantes que consideraban ocupar una posición social alta o media-alta $(34,3 \%)$ y media-baja o baja $(26,8 \%)$. Contrariamente, para aquellos que se consideraban pertenecientes a la clase media el obstáculo que más se oponía a la continuidad de la práctica era la falta de tiempo $(28,8 \%)$ (cuadro 5).

Por último, en los cuadros 6 y 7 se muestran las razones que adujeron 119 de las personas entrevistadas (19\% de la muestra) para no haber practicado nunca actividad física ni deporte en su tiempo libre. Las más habituales fueron la falta de tiempo $(24,4 \%)$ y el hecho de no gustarles hacer ejercicio ni practicar deportes y de no encontrarle utilidad $(24,4 \%)$, seguidas por la edad inadecuada $(18,5 \%)$, la apatía y la falta de hábito $(18,5 \%)$ y los problemas de salud $(8,4 \%)$.

Las pruebas de la $\chi^{2}$ que se realizaron pusieron de manifiesto que las razones señaladas para no haber practi- cado nunca eran independientes del género $\left[\chi^{2}(5)=2,427 ; p=0,799\right.$; IC95\%: $0,791-0,806]$, de la edad $\left[\chi^{2}(20)=19,026\right.$; $p=0,527$; IC95\%: 0,517-0,536] y de la posición social $\left[\chi^{2}(10)=6,132 ; p=0,817\right.$; IC95\%: 0,809-0,825] de los entrevistados.

\section{DISCUSIÓN}

Según se desprende de los resultados de este trabajo, la diversión y la ocupación del tiempo libre, el mantenimiento de la línea y de la forma física, la salud y el gusto por el deporte son, en ese orden, los principales motivos de la muestra estudiada para realizar actividad física en el tiempo libre. Estos datos parecen indicar que en Madrid el hacer ejercicio o practicar deportes es una alternativa elegida para emplear el tiempo libre de forma lúdica y recreativa, dirigida adicionalmente a mantener y mejorar la salud y la imagen física. Estos resultados coinciden con las tendencias observadas para el conjunto de la población española. Según Manuel García, a lo largo de los últimos 30 años la práctica de actividades físico-deportivas ha pasado a ocupar un lugar preferente entre las opciones de tiempo libre y ocio de la población española, evolucionando desde una orientación tradicional, dirigida al rendimiento y a la competición, hacia objetivos rela- 
CUADRO 6. Razón principal para no haber practicado nunca actividad física ni deporte en el tiempo libre, según género y edad. Área metropolitana de Madrid, España, 2005

\begin{tabular}{|c|c|c|c|c|c|c|c|c|}
\hline \multirow[b]{2}{*}{ Razón } & \multicolumn{2}{|c|}{ Género } & \multicolumn{6}{|c|}{ Edad (años) } \\
\hline & $\begin{array}{c}\text { Hombres } \\
(n=32) \\
\% \\
(I C 95 \%)^{\mathrm{a}}\end{array}$ & $\begin{array}{c}\text { Mujeres } \\
(n=87) \\
\% \\
(\mathrm{IC} 95 \%)\end{array}$ & $\begin{array}{c}15-19 \\
(n=6) \\
\% \\
(I C 95 \%)\end{array}$ & $\begin{array}{c}20-29 \\
(n=20) \\
\% \\
(I C 95 \%)\end{array}$ & $\begin{array}{c}30-44 \\
(n=22) \\
\% \\
(I C 95 \%)\end{array}$ & $\begin{array}{c}45-59 \\
(n=37) \\
\% \\
(\mathrm{IC} 95 \%)\end{array}$ & $\begin{array}{c}60-69 \\
(n=34) \\
\% \\
(I C 95 \%)\end{array}$ & $\begin{array}{c}\text { Total } \\
(n=119) \\
\% \\
(\mathrm{IC} 95 \%)\end{array}$ \\
\hline Falta de tiempo & $\begin{array}{c}21,9 \\
(7,6-36,2)\end{array}$ & $\begin{array}{c}25,3 \\
(16,2-34,4)\end{array}$ & $\begin{array}{c}33,3 \\
(0,0-71,0)\end{array}$ & $\begin{array}{c}40,0 \\
(18,5-61,5)\end{array}$ & $\begin{array}{c}27,3 \\
(8,7-45,9)\end{array}$ & $\begin{array}{c}24,3 \\
(10,5-38,1)\end{array}$ & $\begin{array}{c}11,8 \\
(1,0-22,6)\end{array}$ & $\begin{array}{c}24,4 \\
(16,7-32,1)\end{array}$ \\
\hline Edad inadecuada & $\begin{array}{c}18,8 \\
(5,3-32,3)\end{array}$ & $\begin{array}{c}18,4 \\
(10,3-26,5)\end{array}$ & $\begin{array}{l}0,0 \\
\text { NA }\end{array}$ & $\begin{array}{c}5,0 \\
(0,0-14,6)\end{array}$ & $\begin{array}{c}18,2 \\
(2,1-34,3)\end{array}$ & $\begin{array}{c}18,9 \\
(6,3-31,5)\end{array}$ & $\begin{array}{c}29,4 \\
(14,1-44,7)\end{array}$ & $\begin{array}{c}18,5 \\
(11,5-25,5)\end{array}$ \\
\hline $\begin{array}{l}\text { Cansancio, pereza y desgana, falta } \\
\text { de hábito }\end{array}$ & $\begin{array}{c}18,8 \\
(5,3-32,3)\end{array}$ & $\begin{array}{c}18,4 \\
(10,3-26,5)\end{array}$ & $\begin{array}{c}33,3 \\
(0,0-71,0)\end{array}$ & $\begin{array}{c}20,0 \\
(2,5-37,5)\end{array}$ & $\begin{array}{c}18,2 \\
(2,1-34,3)\end{array}$ & $\begin{array}{c}21,6 \\
(8,3-34,9)\end{array}$ & $\begin{array}{c}11,8 \\
(1,0-22,6)\end{array}$ & $\begin{array}{c}18,5 \\
(11,5-25,5)\end{array}$ \\
\hline Problemas de salud, lesiones & $\begin{array}{c}3,1 \\
(0,0-9,1)\end{array}$ & $\begin{array}{c}10,3 \\
(3,9-16,7)\end{array}$ & $\begin{array}{l}0,0 \\
\text { NA }\end{array}$ & $\begin{array}{c}5,0 \\
(0,0-14,6)\end{array}$ & $\begin{array}{c}4,5 \\
(0,0-13,2)\end{array}$ & $\begin{array}{c}8,1 \\
(0,0-16,9)\end{array}$ & $\begin{array}{c}14,7 \\
(2,8-26,6)\end{array}$ & $\begin{array}{c}8,4 \\
(3,4-13,4)\end{array}$ \\
\hline
\end{tabular}

a Intervalo de confianza de $95 \%$.

CUADRO 7. Razón principal para no haber practicado nunca actividad física ni deporte en el tiempo libre, según posición social. Área metropolitana de Madrid, España, 2005

\begin{tabular}{|c|c|c|c|c|}
\hline \multirow[b]{2}{*}{ Razón } & \multicolumn{4}{|c|}{ Posición social } \\
\hline & $\begin{array}{c}\text { Alta/Media-alta } \\
(n=21) \\
\% \\
(\text { IC } 95 \%)^{\mathrm{a}}\end{array}$ & $\begin{array}{c}\text { Media } \\
(n=59) \\
\% \\
(I C 95 \%)\end{array}$ & $\begin{array}{c}\text { Media-baja/Baja } \\
(n=32) \\
\% \\
(\mathrm{IC} 95 \%)\end{array}$ & $\begin{array}{c}\text { Total } \\
(n=119) \\
\% \\
(\mathrm{IC} 95 \%)\end{array}$ \\
\hline Falta de tiempo & $\begin{array}{c}28,6 \\
(9,3-47,9)\end{array}$ & $\begin{array}{c}27,1 \\
(15,8-38,4)\end{array}$ & $\begin{array}{c}18,8 \\
(5,3-32,3)\end{array}$ & $\begin{array}{c}24,4 \\
(16,7-32,1)\end{array}$ \\
\hline No le gusta, no le ve beneficios ni utilidad & $\begin{array}{c}23,8 \\
(5,6-42,0)\end{array}$ & $\begin{array}{c}18,6 \\
(8,7-28,5)\end{array}$ & $\begin{array}{c}28,1 \\
(12,5-43,7)\end{array}$ & $\begin{array}{c}24,4 \\
(16,7-32,1)\end{array}$ \\
\hline Edad inadecuada & $\begin{array}{c}19,0 \\
(2,2-35,8)\end{array}$ & $\begin{array}{c}25,4 \\
(14,3-36,5)\end{array}$ & $\begin{array}{c}9,4 \\
(0,0-19,5)\end{array}$ & $\begin{array}{c}18,5 \\
(11,5-25,5)\end{array}$ \\
\hline Cansancio, pereza y desgana, falta de hábito & $\begin{array}{c}14,3 \\
(0,0-29,3)\end{array}$ & $\begin{array}{c}16,9 \\
(7,3-26,5)\end{array}$ & $\begin{array}{c}25,0 \\
(10,0-40,0)\end{array}$ & $\begin{array}{c}18,5 \\
(11,5-25,5)\end{array}$ \\
\hline Problemas de salud, lesiones & $\begin{array}{c}9,5 \\
(0,0-22,0)\end{array}$ & $\begin{array}{c}6,8 \\
(0,4-13,2)\end{array}$ & $\begin{array}{c}12,5 \\
(1,0-24,0)\end{array}$ & $\begin{array}{c}8,4 \\
(3,4-13,4)\end{array}$ \\
\hline Otras & $\begin{array}{c}4,8 \\
(0,0-13,9)\end{array}$ & $\begin{array}{c}5,1 \\
(0,0-10,7)\end{array}$ & $\begin{array}{c}6,3 \\
(0,0-14,7)\end{array}$ & $\begin{array}{c}5,9 \\
(1,7-10,1)\end{array}$ \\
\hline
\end{tabular}

a Intervalo de confianza de $95 \%$.

cionados principalmente con la diversión, la estética y la salud $(5,31)$.

$\mathrm{Al}$ igual que apuntan otros estudios $(4-6,11,14)$, los motivos de práctica de los madrileños difieren en función de su edad y género. Respecto al género, los hombres parecen practicar, sobre todo, por diversión y ocupación del tiempo libre, mientras que las mujeres lo hacen mayoritariamente por mantener la forma y la línea. Estos resultados observados en las mujeres coinciden con los hallados en diferentes estudios de características similares, tendiendo a manifestar con mayor frecuencia motivos de práctica relacionados con aspectos estéticos, concretamente con el control del peso corporal y con la imagen $(5-7,9,10)$. Tales hallazgos, no obstante, no concuerdan con los presentados en otros trabajos, cuyos autores informan que el ejercicio físico en los hombres, a diferencia de las mujeres, tiene una orientación más dirigida a la competición y al desarrollo de habilidades y capacidades propias del deporte tradicional (11).

Por grupos de edad, los motivos para realizar actividades físicas o practicar de- portes manifestados por los habitantes del área metropolitana de Madrid siguen una tendencia idéntica a la presentada por el conjunto de la población española (5). Así, a medida que avanza la edad de los entrevistados, los motivos aludidos van cambiando de orientación. Entre los más jóvenes predomina inicialmente la diversión y la ocupación del tiempo libre como motivo principal, virando hacia el cuidado de la forma física y de la línea en los adultos y en los adultos mayores, así como hacia el mantenimiento y la mejora de la salud entre estos últimos. Práctica- 
mente todos los trabajos revisados informan tendencias similares $(6,7,14)$ que por lógica obedecen a las diferentes aspiraciones, objetivos, metas y necesidades que poseen cada uno de los grupos de población.

Desde el ángulo de la posición social, los análisis efectuados muestran que no existen relaciones entre esta variable y los motivos argumentados por los practicantes para realizar actividad física y/o deporte. Incluso más, los resultados del presente estudio también nos permiten afirmar que las personas de la zona metropolitana de Madrid que han dejado de realizar actividad física o deporte en su tiempo libre, lo han hecho empujadas principalmente por las exigencias derivadas del trabajo, de los estudios o de la familia, por la falta de tiempo o por problemas de salud y lesiones. La primera de estas razones para el abandono de la práctica coincide con la hallada para el conjunto de la población española (5). Dicho esto, sí se han observado diferencias en el resto de motivos, al presentarse la pereza y la desgana, junto con los problemas de salud y la edad, como los siguientes determinantes de abandono entre los españoles. Es posible que los grandes trayectos entre el hogar y el lugar de estudio o de trabajo, las largas esperas y otros obstáculos de la vida en las grandes ciudades puedan contribuir a explicar por qué entre los habitantes de Madrid la escasez de tiempo es un factor más limitante que para el resto de la población española, tal como se observa en este y otros estudios $(1,6,21)$.

Además, al analizar los motivos por los que una parte de los entrevistados nunca habían practicado actividad física ni deportes en su tiempo libre, se detectó que una de cada cuatro personas aludió, de nuevo, la falta de tiempo y, una proporción idéntica, que no le veía beneficios ni utilidad. Estas razones coinciden en buena medida con las encontradas en otros estudios realizados en períodos y contextos sociales similares. En uno de ellos, por ejemplo, la Comisión Europea indica que el $34 \%$ de los europeos inactivos durante su tiempo libre argumentaban la falta de tiempo para justificar su comportamiento, mientras que otro $25 \%$ señalaban el hecho de no gustarles hacer ejercicio y practicar deportes (30). Del mismo modo, otros estudios efectuados fuera del contexto europeo también parecen indicar que estas razones son los principales argumentos para no haber practicado nunca actividad física ni deporte $(6,22,34)$.

Si bien hay estudios que indican que las mujeres parecen percibir un mayor número de impedimentos que los hombres para realizar actividades físicas en su tiempo libre $(2,18)$, en el presente, y en otros estudios (1), se ha observado que las razones para haber abandonado el ejercicio o el deporte en el tiempo libre, o para no haberlos practicado nunca, se distribuyen de manera muy similar entre hombres y mujeres. La única diferencia que cabe señalar en este sentido es que la edad inadecuada, aunque sin ser la razón más importante para abandonar la práctica, sí parece constituir un factor más determinante para esta conducta en los hombres que en las mujeres.

En relación con la edad y las razones para haber abandonado la actividad física o el deporte, los resultados obtenidos muestran que en el caso de los madrileños y madrileñas más jóvenes (15 a 19 y 20 a 29 años), la falta de tiempo es claramente el impedimento primordial, aunque disminuyendo su protagonismo a medida que aumenta la edad. De este modo, entre los adultos de mayor edad (45 a 69 años) esta razón pierde relevancia, cediendo lugar a las obligaciones laborales y familiares, la edad excesiva y los problemas de salud y las lesiones. Estas observaciones son compartidas por otros autores. En un estudio sobre una muestra de 2298 adultos australianos se halló que para los más jóvenes, la falta de tiempo, la pérdida de motivación y el cuidado de los hijos eran las razones más frecuentemente citadas para el abandono de la práctica, en tanto que para el grupo de mayor edad, lo eran las lesiones y la falta de salud. Además, en este último grupo, una de cada cinco personas manifestó ser demasiado mayor para hacer ejercicio (1).

A la vista de estos resultados pueden realizarse dos reflexiones. Por un lado, el hecho de que los grupos de población de mayor edad (de 60 a 69 años y, especialmente, de 45 a 59 años) crean tener demasiada edad para continuar con la actividad física, en muchos casos podría estar relacionado con el desconocimiento de las distintas opciones que hay para poder hacer ejercicio o practicar deportes acordes con sus posibilidades y características específicas. Por el otro, resulta paradójico que los adultos mayores (60 a 69 años) señalen la edad como el principal impedimento para la actividad física, aun cuando justamente el mismo grupo ha expresado que el cuidado de la salud es también la principal razón para hacer ejercicio o practicar deportes. Adicionalmente, vale decir que la posición social, al igual que ocurría en el caso de las razones para realizar actividades físicas en el tiempo libre, tampoco parece tener una influencia directa en las causas para haber abandonado la práctica o para no haber practicado nunca, resultados que coinciden con los hallados para el conjunto de la población española (5).

La principal limitación del presente estudio podría radicar en la validez externa de algunos de sus resultados. En efecto, el tamaño de la muestra en el caso de los entrevistados que declararon haber abandonado la actividad física en su tiempo libre $\mathrm{y}$, particularmente, el menor número de personas que manifestaron no haber practicado nunca actividad física, pueden hacer que los resultados relativos al abandono de la práctica y, sobre todo, a las razones que justifican no haber realizado nunca actividad física ni deporte, no resulten del todo precisos, máxime cuando se desagregan en función del género, la posición social o la edad de los participantes. Así pues, en futuros estudios sería interesante seleccionar una muestra de mayor tamaño que permitiera profundizar en los factores analizados.

En conclusión, puede decirse que la práctica físico-deportiva en la muestra estudiada tiene un carácter lúdico y recreativo, además de estar dirigida al cuidado de la salud y a la adquisición de un buen aspecto físico. La diversión y la ocupación del tiempo libre son el principal motivo que conduce con mayor frecuencia a los hombres a realizar a hacer ejercicio o practicar deportes, mientras que en las mujeres es más habitual el mantenimiento de la forma o de la línea. Puede verse que las razones para practicar también evolucionan con la edad, dado que mientras que en los adolescentes y los jóvenes priman la diversión y la ocupación amena del tiempo, y en los adultos cobra mayor importancia el mantenimiento de la forma y de la línea, entre los adultos mayores, el mantenimiento o la mejora de la salud son las motivaciones principales para realizar actividades físicas. Las exigencias laborales y las obligaciones familiares, la falta de tiempo y los problemas de salud y las lesiones, son las causas más frecuentes para el abandono de la práctica por parte de los entrevistados. La falta de tiempo destaca principalmente como 
motivo de abandono entre adolescentes y jóvenes, en tanto que para los adultos de más edad (45 años en adelante) lo son las obligaciones laborales y familiares, la edad excesiva y los problemas de salud y las lesiones. Por último, el hecho de no verle beneficios ni utilidad y, de nuevo, la falta de tiempo, son las principales razones que argumentan los entrevistados para no haber practicado nunca, independientemente de su género, edad y posición social.

En consideración de todo lo expuesto, y con el objeto de orientar futuras estrategias de intervención para promover el ejercicio físico y la práctica de deportes, sobre todo entre los grupos de población en los que se registran menores niveles de actividad físico-práctica (adultos y mayores, mujeres y personas de posición social media-baja o baja), se presentan las siguientes recomendaciones:

- Potenciar programas de actividades físicas y deportivas dirigidos a manteical activity preferences, preferred sources of assistance, and perceived barriers to increased activity among physically inactive Australians. Prev Med. 1997;26(1):131-7.

2. Cohen-Mansfield J, Marx MS, Guralnik JM. Motivators and barriers to exercise in an older community-dwelling population. J Aging Phys Activ. 2003;11(2):242-53.

3. Sheppard L, Senior J, Park CH, Mockenhaupt R, Chodzko-Zajko W. Strategic priorities for increasing physical activity among adults age 50 and older: The national blueprint consensus conference summary report. J Aging Phys Activ. 2003;11(3):286-92.

4. Burton NW, Turrell G, Oldenburg B, Sallis JF. The relative contributions of psychological, social, and environmental variables to explain participation in walking, moderate-, and vigorous-intensity leisure-time physical activity. J Phys Activ Health. 2005;2(2):181-96.

5. García M. Postmodernidad y deporte: entre la individualización y la masificación. Encuesta sobre hábitos deportivos de los españoles, 2005. Madrid: Consejo Superior de Deportes y Centro de Investigaciones Sociológicas; 2006.

6. Ruiz F, García ME, Díaz A. Análisis de las motivaciones de práctica de actividad física y de abandono deportivo en la ciudad de La Habana (Cuba). Anales de Psicología. 2007;23(1): $152-66$.

7. Monteiro CA, Conde WL, Matsudo SM, Matsudo VR, Bonseñor IM, Lotufo PA. A descriptive epidemiology of leisure-time physical activity in Brazil, 1996-1997. Rev Panam Salud Publica. 2003;14(4):246-54. ner y mejorar la salud y a adquirir una buena forma física, pero que utilicen como base de actuación el componente lúdico y recreativo de este tipo de actividades para tratar de lograr los mayores niveles posibles de participación y adherencia.

- Acercar la práctica de la actividad física y el deporte al lugar de trabajo o de residencia, con el fin de minimizar la "pérdida de tiempo" por posibles desplazamientos al lugar habitual de práctica. Con esta idea, podrían establecerse mecanismos que incentivaran el desarrollo de programas de actividad física y deporte en las propias empresas o centros de trabajo, y que promovieran aún más la práctica físico-deportiva en la escuela. Asimismo, debería apoyarse la creación de instalaciones deportivas en lugares que todavía presenten carencias de ciertas tipologías de equipamientos deportivos, al igual que en los centros educativos e incluso en los propios centros de trabajo.

\section{REFERENCIAS}

8. Capdevila L, Niñerola J, Cruz J, Losilla JM, Parrado E, Pintanel M, et al. Exercise motivation in university community members: A behavioural intervention. Psicothema. 2007; 19(2):250-5.

9. Anderson $C B$. When more is better: number of motives and reasons for quitting as correlates of physical activity in women. Health Educ Res. 2003;18(5):525-37.

10. Foster C, Hillsdon M, Cavill N, Allender S, Cowburn G. Understanding participation in sport: A systematic review. London: Sport England; 2005

11. Beggs BA, Stitt JE, Elkins DJ. Leisure of participation and nonparticipation in campus recreational sports programs. Recreational Sports Journal. 2004;28(1):65-77.

12. Clark DO. Identifying psychological, physiological, and environmental barriers and facilitators to exercise among older low income adults. Journal of Clinical Geropsychology. 1999;5(1):51-62

13. Wilcox S, Bopp M, Oberrecht L, Kammermann SK, McElmurray CT. Psychosocial and perceived environmental correlates of physical activity in rural and older African American and White women. J Gerontol B Psychol Sci Soc Sci. 2003;58(6):329-37.

14. Allender S, Cowburn G, Foster C. Understanding participation in sport and physical activity among children and adults: a review of qualitative studies. Health Educ Res. 2006; 21(6):826-35.

15. Grossman MD, Stewart AL. "You aren't going to get better by just sitting around": Physical activity perceptions, motivations, and barriers
- Realizar una labor eficaz de información orientada a la población general $\mathrm{y}$, en especial, a las de mayor edad, sobre los beneficios que tiene para la salud adoptar un estilo de vida activo, incluyendo la práctica regular de actividad física o deporte. También se debería informar a la gente respecto a las innumerables posibilidades que existen para poder desarrollar una práctica acorde con las características de cada persona, incluidas la edad y la condición física.

- Ofrecer alternativas en los centros deportivos o en los lugares de trabajo que permitan conciliar la práctica de la actividad física y los deportes con las responsabilidades familiares o laborales.

Agradecimientos. Este estudio ha sido promovido y financiado por la Asociación Madrileña de Empresarios Deportivos (ASOMED) y por la Dirección General de Promoción Deportiva de la Comunidad de Madrid. in adults 75 years of age or older. Am J Geriatr Cardiol. 2003;12(1):33-7.

16. Cecchini JA, Méndez A, Muñiz J. Motives for practicing sport in Spanish schoolchildren. Psicothema. 2002;14(3):523-31.

17. Balde A, Figueras J, Hawking DA, Miller JR. Physician advice to the elderly about physical activity. J Aging Phys Activ. 2003;11(1):90-7.

18. Kendzierski D, Johnson W. Excuses, excuses, excuses: a cognitive behavioral approach to exercise implementation. J Sport Exerc Psychol. 1993;15(2):207-19.

19. O'Dea JA. Why do kids eat healthful food? Perceived benefits of and barriers to healthful eating and physical activity among children and adolescents. J Am Diet Assoc. 2003;103(4): 497-504.

20. Felton GM, Boyd MD, Bartoces MG, Tavakoli AS. Physical activity in young African American women. Health Care Women Int. 2002; 23(8):905-18.

21. Parks SE, Housemann RA, Brownson RC. Backgrounds in the United States and rural adults of various socioeconomic Differential. J Epidemiol Community Health. 2003;57(1): 29-35.

22. Myers RS, Roth DL. Perceived benefits of and barriers to exercise and stage of exercise adoption in young adults. Health Psychol. 1997;16(3):277-83.

23. Satariano WA, Haight TJ, Tager IB. Reasons given by older people for limitation or avoidance of leisure time physical activity. J Am Geriatr Soc. 2000;48(5):505-12.

24. Rasinaho M, Hirvensalo M, Leinonen R, Lintunen T, Rantanen T. Motives for and barriers 
to physical activity among older adults with mobility limitations. J Aging Phys Activ. 2006; 15(1):90-102.

25. Garber CE, Blissmer BJ. The challenges of exercise in older adults. En: Burbank PM, Riebe D, eds. Promoting exercise and behavior change in older adults: Interventions with the transtheoretical model. New York: Springer; 2002. Pp. 29-56.

26. Allison KR, Dwyer JJ, Goldenberg E, Fein A, Yoshida KK, Boutilier M. Male adolescents reasons for participating in physical activity, barriers to participation, and suggestions for increasing. Adolescence. 2005;40(157):155-70.

27. Cress ME, Buchner DM, Prohaska T, Rimmer J, Brown M, Macera C, et al. Best practices for physical activity programs and behavior counseling in older adult populations. J Aging Phys Activ. 2005;13(1):61-74.

28. Rodríguez-Romo G, Mayorga JI, Merino A, Garrido M, Fernández M. Hábitos deportivos de la población de la Comunidad de Madrid, 2005. Madrid: Dirección General de Deportes-Consejería de Cultura y Deportes de la Comunidad de Madrid; 2005.

29. Rodríguez-Romo G, Mayorga JI, Merino A, Garrido M, Fernández M. La práctica, el abandono y la demanda futura de actividad física y/o deporte entre los habitantes de la Comunidad de Madrid. Kronos. 2006;5(9):54-66.

30. European Commission. The citizens of the European Union and Sport. Hallado en: http:// ec.europa.eu/public_opinion/archives/ebs/ ebs_213_summ_en.pdf. Acceso el 20 de agosto de 2009.

31. García M. Los españoles y el deporte: prácticas y comportamientos en la última década del siglo XX. Encuesta sobre los hábitos deportivos de los españoles, 2000. Madrid: Ministerio de Educación Cultura y DeporteConsejo Superior de Deportes; 2001.
32. Baranda L. Enquesta sobre la pràctica d'activitats físico-esportives a Catalunya. Barcelona: Secretaria General de l'Esport; 1995.

33. García M, Mestre JA. Los hábitos deportivos de la población de Valencia (2000). Valencia: Ayuntamiento de Valencia-Fundación Deportiva Municipal; 2002.

34. Seclén-Palacín JA, Jacoby ER. Factores sociodemográficos y ambientales asociados con la actividad física deportiva en la población urbana del Perú. Rev Panam Salud Publica. 2003;14(4):255-64.

Manuscrito recibido el 25 de julio de 2008. Aceptado para publicación, tras revisión, el 5 de febrero de 2009.

ABSTRACT Objectives. To analyze the motivators and barriers to physical activity and sports among the population of metropolitan Madrid, as well as any correlations with gender, age, and social status.

\section{Reasons for and barriers to exercising and sports participation in Madrid}

Methods. A cross-sectional study of a sample of 625 individuals whose data were representative of the whole population, with a $\pm 4 \%$ margin of error and a $95.5 \%$ confidence interval $(95 \% \mathrm{CI})$. The sampling type selected was multistage, utilizing an ad hoc questionnaire.

Results. The most frequently cited motivations for exercising were: for fun (29.9\%), to stay in shape $(26.4 \%)$, and health $(16.1 \%)$, differing according to gender $\left(\chi^{2}(8)=19.635\right.$; $P=0.009$; 95\%CI: $0.007-0.011)$ and age $\left(\chi^{2}(32)=55.671 ; P=0.006\right.$; 95\%CI: $\left.0.005-0.0080\right)$. With regard to activity cessation, the demands of work and family $(24.1 \%)$ and lack of time $(22.2 \%)$ were the most common reasons, correlating with age $\left(\chi^{2}(24)=36.955\right.$; $P=0.041 ; 95 \%$ CI: $0.037-0.044)$ and slightly with gender $\left(\chi^{2}(6)=11.753 ; P=0.069 ; 95 \%\right.$ CI: $0.063-0.074)$. The reasons most often cited for never exercising were lack of time $(24.4 \%)$ and not enjoying it (24.4\%), uncorrelated with any of the study variables.

Conclusions. Among the study sample, the purpose of sports and exercise was for fun and recreation, aimed at maintaining and improving health and acquiring an attractive figure. Motivators driving activity and discontinuation were correlated with age and gender. Conversely, the reasons for never exercising were independent of the study variables.

Key words Physical activity; exercise; motivation; sports; life style; health behavior; Spain. 